\title{
Ways in which healthcare organisations can support overseas-qualified doctors in the UK
}

\author{
Charlotte Rothwell, Gill Morrow, Bryan Burford, Jan Illing \\ Centre for Medical Education Research, Durham University, School of Medicine, Pharmacy and Health, UK \\ Correspondence: Charlotte Rothwell, Research Associate, Centre for Medical Education Research, Durham University, School of \\ Medicine, Pharmacy and Health, UK. Email: c.r.rothwell@durham.ac.uk
}

\begin{abstract}
Objectives: To identify what healthcare organisations, including medical regulators, can do to address the issues and concerns faced by overseas-qualified doctors when moving to the UK.

Methods: Qualitative interviews were conducted with sixtysix doctors who had qualified outside the UK and who were entering the first year of the UK Foundation Programme (FY1, equivalent to the intern year). Doctors were interviewed three times at different points in that year. In addition twelve telephone interviews were conducted with educational supervisors. A grounded theory approach was used to analyse the findings.
\end{abstract}

Results: Issues and concerns were found to relate to: practi- cal and logistical difficulties; gaps in declarative knowledge relevant to the UK, for example, use of equipment and drug names; structural differences, for example, organisational elements of working in the NHS, and differences in the relational aspects of professional work and clinical care, stemming from training in different models of healthcare.

Conclusions: Agencies at different levels - government, regulator and employer - have a role to play in supporting overseas-doctors in their transition, and it is suggested that a co-ordinated approach would have benefits for doctors and patients.

Keywords: Overseas doctors, international medical graduates, support, medical education, induction programmes

\section{Introduction}

The healthcare workforce is becoming more international and globalised. ${ }^{1}$ Migration of medical staff is not new, the National Health Service (NHS) in the UK, in common with other countries, has historically relied on overseas-qualified staff to ensure it can effectively deliver healthcare. In 2010, $37 \%$ of the doctors registered with the UK medical regulator (the General Medical Council [GMC]) qualified in other countries. ${ }^{2}$ While there is a downward trend with increased output from UK medical schools and restrictions on visa requirements ${ }^{3,4}$ overseas-qualified doctors are still entering the UK workplace. At the same time there are concerns that overseas-qualified doctors are over-represented in cases about doctors' performance which come to the attention of the GMC and/or the National Clinical Assessment Service (NCAS). $)^{5-7}$

Doctors coming from overseas face not only changes in their personal and domestic lives, but also professionally. Healthcare systems and regulatory frameworks in different countries may have different values and expectations, causing difficulties when practising. ${ }^{8}$ Regulators in other countries may focus more on the interests and welfare of doctors than on patients and quality of care. ${ }^{9}$

There is a substantial literature looking at the adjustment of migrant workers to a new country - in a number of business domains. ${ }^{10}$ Much work is based on the identification of three elements of transition; general adjustment, work adjustment and interaction adjustment. ${ }^{11-13}$

\footnotetext{
"General [cultural] adjustment - comfort associated with various non-work factors such as general living conditions, local food, transportation. Work adjustment - comfort with the assigned job or tasks. Interaction adjustment - comfort associated with interaction with host-country nationals both inside and outside work" ${ }^{13}$
}

Studies in healthcare have looked at different elements of the transition, some specific to the workplace, some more general. These issues are discussed in more detail below. Examples of general adjustment include the practicalities of moving to a new country, including immigration and professional registration requirements. ${ }^{3}$ 
Financial issues in the period between arrival in a country and starting work may lead to more practical concerns such as finding suitable accommodation. There can also be cultural issues including feelings of social isolation and cultural disorientation. ${ }^{15-19}$ Interaction adjustment may emerge in subtle ways, such as unfamiliarity with nonverbal communication, ${ }^{8,20,21}$ which may also present themselves in work-specific settings. Lack of professional and personal networks can make integration and adaptation into the NHS difficult. ${ }^{22}$ Differences in training cultures may lead to difficulties adapting to hierarchies and different relationships with teachers and seniors. ${ }^{16,20,23}$

Difficulties may arise around the colloquial expressions used by patients ${ }^{14,24,25}$ and jargon and abbreviations used in the clinical setting, ${ }^{24,26}$ leading to confusion, misunderstandings and potential risks to patient safety. A relative lack of language skills may be interpreted as indicating a lack of medical knowledge and skills. ${ }^{16}$ Communicating emotional support for patients can be a challenge for overseasqualified doctors, for example across culture or gender. ${ }^{27,28}$ Explicit work adjustment difficulties have been identified in the way healthcare is structured. The UK healthcare system operates in a less hierarchical way than many others ${ }^{16}$ with multi-disciplinary team-working an important element. However this can be a challenge to overseas doctors from more hierarchically-oriented cultures ${ }^{29}$ where there is greater demarcation between professions. Understanding of roles and responsibilities of other team members can also be lacking. ${ }^{16,25}$

The UK emphasises a patient-centred approach where patients are more involved in their care, ${ }^{30}$ which may be at odds with the values and experience doctors bring from other countries. ${ }^{31}$ It can be the norm for doctors to discuss a patient's illness and treatment with relatives before the patient. ${ }^{16}$ Overseas doctors may have little experience of patients asking questions about treatment options or questioning the doctor's authority. ${ }^{8,20}$

There are efforts to help doctors make the transition to new countries. Some deaneries (Deaneries are the bodies responsible for postgraduate medical education at regional level in the UK) already offer specific induction courses targeted at overseas doctors, but the GMC are piloting an induction programme for all doctors new to working in the NHS. $^{32}$

\section{The current study}

The current study looked at the experience of overseasqualified doctors starting the first year of the UK's Foundation Programme in 2007. Foundation Programme is the two year postgraduate programme (FY1 and FY2) which all new doctors in the UK must complete before moving on to specialty training (equivalent to the intern level in other systems). The study considered the differences identified by overseas-qualified doctors as they began and progressed through FY1, and what factors helped or hindered the ease of their transition to the UK workplace. ${ }^{33}$ The paper draws on findings from a larger study on the experiences of UK, European Union (EU) (non-UK) and non-EU medical graduates making the transition into the UK workplace.

\section{Aim of paper}

This paper aims to identify what healthcare organisations, including medical regulators, can do to address the issues and concerns faced by overseas-qualified doctors when moving to the UK.

\section{Methods}

The study was approved by the NHS Research Ethics Service (Cambridgeshire 1 Research Ethics Committee). Overseas-qualified doctors entering FY1 were recruited from five deaneries in the UK. Participants were sent an information sheet and letter of invitation by email from Postgraduate Deaneries in the UK. Participants volunteered by direct contact with the research team.

Participants who responded to the invitation were interviewed by telephone three times: at the beginning of FY1, at the end of their first placement (4 months) and finally at the end of FY1 (12 months). The interviews were conducted by the four authors and lasted 30-60 minutes. Educational supervisors who had worked with some of these graduates as FY1s were also invited, via e-mail, to take part in one telephone interview (20-30 minutes) to provide triangulation on the trainees' self-perceptions. Interviews were recorded with participants' permission and transcribed verbatim.

\section{Interview schedule}

Questions for the semi-structured interview were developed from a literature review and pilot interviews with five overseas-qualified doctors already on the Foundation Programme. The questions covered broad areas (preparedness for practice, communication, team-working and factors that helped or hindered the transition to the UK workplace). The follow-up interview schedules were developed following analysis of the initial interviews. In the second and third interview researchers referred back to issues discussed in previous interviews to aid continuity of discussion and to serve as member checking (respondent validation).

\section{Analysis}

The analysis took a constructivist, grounded theory approach. ${ }^{34}$ This assumes that researchers and participants construct meaning through the interview and analysis process, reflecting important elements of the participants' experience, and new meaning is constructed from the data and the researcher. Transcripts were coded using the NVivo 7 software package. ${ }^{35}$ Themes including the experience of transition, communication, and clinical practice, experience of working as a doctor in the UK and factors which had helped and hindered transition were identified through 
review of the data and discussion between the research team. Further analysis identified four overarching analytical themes, classifying the types of issue which were identified: practical and logistical problems, gaps in knowledge, structural differences and relational differences.

\section{Findings}

Table 1 gives the number of interviews conducted with trainees in each stage. Sixty-six overseas doctors were initially recruited to the study. A total of 152 interviews with trainees took place (64 initial interviews, 56 at first followup and 32 at second follow-up). Every effort was made to contact participants and in some cases contact details had changed or interviews were unable to be carried out in the target period of the study. However the researchers agreed that data saturation across the analytical themes had been achieved. In addition a total of twelve telephone interviews were conducted with Educational Supervisors. IDs were given to quotes in the text as follows: initial interviews (EU/non-EU a), first follow-up (EU/non-EU b), second follow-up (EU/non-EU c) and Educational Supervisors (ES).

Table 1. Total number of interviews completed with overseas doctors

\begin{tabular}{lccc}
\hline Location & Initial interviews & $\begin{array}{c}\text { Follow-up at 4 } \\
\text { months }\end{array}$ & $\begin{array}{c}\text { Follow-up at 12 } \\
\text { months }\end{array}$ \\
\hline Non-EU & 52 & 45 & 27 \\
EU (non-UK) & 12 & $\begin{array}{c}11 \\
(2 \text { new recruits) }\end{array}$ & 5 \\
Total & 64 & 56 & 32 \\
\hline
\end{tabular}

Participants came from fourteen non-EU countries and six EU countries (these were mostly, at the time, new member countries). Table 2 shows the countries represented in the study. Twenty-nine participants were female, 37 male. Participants had been in the UK between one day and eight years when initially interviewed, with a mode of two years. Period since qualification ranged from a few months to ten years (with a mode of twelve months). Some had limited previous experience of the NHS through undergraduate elective placements or postgraduate clinical attachments (periods of observation rather than clinical practice in the NHS). Fourteen of the participants had attended no induction or shadowing prior to starting their FY1 post.

\section{Findings from interviews}

Findings are discussed under the overarching analytical themes identified in analysis. Certain issues necessarily overlapped more than one theme.

\section{Practical and logistical difficulties on arrival}

Some practical difficulties occurred in relation to the process of immigration such as dealing with visa requirements, and GMC registration. Practical difficulties after arrival in the UK were identified in everyday requirements such as banking, driving licences and finding schools for children.

Table 2. Countries represented in the study

\begin{tabular}{ll}
\hline EU & Non-EU \\
\hline Austria & Afghanistan \\
Italy & Bangladesh \\
Lithuania & Cuba \\
Malta & Egypt \\
Poland & India \\
Romania & Iraq \\
& Jordan \\
& Nigeria \\
& Pakistan \\
& Russia \\
& Sierra Leone \\
& Sudan \\
& Syria \\
& United Arab Emirates \\
\hline
\end{tabular}

Information that doctors would have liked to receive was mostly general information about living in the UK. Several participants commented on practical ways in which their transition to the UK workplace could have been improved, including greater efficiency in organising work permits; help with setting up a bank account and other financial arrangements, gaining internet access and clarity on health tests required for entry. A timeline of applying for the Foundation Programme would also have been useful for some.

\footnotetext{
"I struggled with having a bank account and having a mobile phone, with having internet access... I said I came from abroad and 'Well we can't give you a bank account if you don't have previous accounts'...”. (14c, non-EU)

"...how to get your mortgages and rent and all this kind of stuff sorted out, maybe that's something they could have some guidance about...[that] covers their out-of-hospital worries". (17c, non-EU)
}

Both educational supervisors and overseas doctors recognised that help with practical issues outside of work would aid in doctors settling more easily at work.

\footnotetext{
"They may well arrive with wives, sometimes with children as well...so they've got to get used to a new country, culture, healthcare settings, but also settle families as well...you won't be happy in your job unless you're happy outside". (ES9)

"So far all the hospital is interested in is the work inside the hospital... They don't concentrate a lot what happens outside, they think it's not their problem but I think it all comes together to be able to function fully in the hospital". (3c, non-EU)
}

Many participants commented that support outside work from friends and family, especially those who worked in the NHS themselves, was very helpful for dealing with practical difficulties, as well as being able to discuss concerns and 
queries. Family and friends also provided direct practical help such as financial support and accommodation.

\section{Gaps in declarative knowledge}

There were some gaps in declarative knowledge, for example, simple statements of fact or pieces of information to which the doctors had not been previously exposed. Clinical areas identified were around policy and legal requirements (including how to complete a death certificate), handover systems, knowledge of some equipment (such as scanners), and prescribing. Although the science is the same, names used for drugs varied - some participants learnt using chemical names, while generic names are used throughout the NHS, and brand names may be used with patients. On a procedural level, the routine use of the British National Formulary (BNF) was new to many, although some doctors had come across it before starting FY1.

"I think a little bit more introduction about the general principles and ethical systems". (ES18)

\section{Structural differences}

Some differences related to the organisation of the NHS and professional relationships. Doctors may be told about these before starting work, but there was a sense that the procedural knowledge of these relationships could only be developed with an understanding of the context. The majority of participants felt they had good knowledge of their own role and responsibilities before starting as an FY1, from information obtained from websites and job descriptions, although some would have liked to have received this information earlier. There were expectations that teams in the UK would be more comprehensive and team working would be more organised.

For some, working in a multi-professional team was a novelty. Some participants were not clear about roles of senior doctors, different grades of nurse, allied health professionals and non-clinical professions such as social workers. Some also felt they were initially lacking in understanding of the relationship between primary and secondary care in the UK, as there is not such a clear distinction in some other systems.

"I wasn't quite familiar with the competencies of every team member that was in my team. For example, I wasn't quite sure what a nurse can do, for example if she can put an IV cannula in or if she can give IV antibiotics... if I should do it myself or should I just request it ...." (22b, EU)

"Pharmacists and nurses, I don't believe I had quite a clear idea about their responsibilities...nurses I knew afterwards that they arrange for social work...I didn't know exactly how they do it ... But about the other senior members of the team like the F2 or the registrar and the consultant I think I had a good idea...because our consultant explained". (3b, non-EU)
Differences in prescribing practice were also identified. In some countries senior doctors take responsibility for prescribing, and juniors may not prescribe controlled drugs. There was uncertainty about who can and cannot prescribe in the UK.

Experiences before starting work, such as on clinical attachments, had been invaluable in understanding the NHS, but the process of actually finding an attachment was difficult. The Professional and Linguistic Assessments Board (PLAB) exams taken by non-EU doctors were found to be very beneficial in increasing confidence.

“...passing it [PLAB exam] in itself is like getting you very much prepared for it". (43a, non-EU)

Structural differences between systems and roles had been addressed in induction (generally a day or more of structured teaching and information introducing the doctor to their workplace) and shadowing (where the new doctor follows a working F1 in the workplace). Shadowing is part of the Foundation Programme induction process, but its precise implementation varies with locality. Some participants had attended an induction programme specifically targeted at overseas doctors to address their particular needs.

\footnotetext{
"We knew before starting the job what the system was and what was expected from us because they did an overseas induction in [the Trust]". (26b, non-EU)
}

While shadowing was helpful, it was not always long enough to provide sufficient learning opportunities and its effectiveness was felt to be dependent upon who was shadowed and the opportunities available. Several participants said they had missed their induction and shadowing period, mainly due to complications with visas and paperwork, which had delayed their entry into the UK.

\section{Relational differences}

The final and most far-reaching area of difference was in the way relationships with patients and other staff are conducted. The use of language also arose here - while vocabulary may be understood, the appropriateness of vernacular language, and how language is used socially, and at sensitive times, is a more sophisticated cultural skill. Some participants also noted the role of non-verbal communication, particularly during patient interactions, such as the appropriateness of making eye contact which is not the same in some cultures.

\section{Relationships with patients}

The patient-centred approach was new to many participants and particularly apparent in areas such as obtaining patient consent, and the extent to which patients are made aware of their condition and involved in their care. 
Often in doctors' home countries, relatives were told about illness first, and the decision whether and how much to tell the patient placed with them. Patients in the UK were felt to be more knowledgeable and better informed about their illnesses, compared to other countries where patients often did not - or were not expected to want to - know what was wrong with them.

"In the UK you need to explain all the things what you will have to do... and before you put a cannula you have to, "Oh this is a cannula we will put in your vein because we will give you some drip", And in [EU country] it is really much quicker...we don't have to explain what it is and why..." (64a, EU)

\section{Relationships with other staff}

Relational differences were also observed in relationships with other staff. Some participants reported that the health system they had trained or worked in before was very hierarchical, both within the medical profession and between professions, whereas in the UK hierarchies are flatter and more fluid. Nurses were not seen as colleagues in some systems, whereas in the UK they may have more specialised and skilled roles, and are a part of the clinical team whose opinion can be valued.

“...the relationship is important and the nurses are like colleagues and like team members [in the UK]... [in country] there are still barriers". (30a, non EU)

Relationships with senior doctors were also different, with senior doctors being more approachable and friendly in the UK. Calling a senior by his/her first name is often expected, whereas it would be seen as disrespectful elsewhere. Approaching seniors to ask for help is strongly encouraged in the UK, including at night, but would not always be acceptable elsewhere.

\begin{abstract}
"Back home...there is a kind of respect like you are not allowed to speak to someone who is believed to be your senior easily... the culture does not allow you to speak to people who are older than you the way you want to". (35a, non-EU)
\end{abstract}

This was also noted by educational supervisors - all senior doctors - who reported that overseas doctors could appear subservient or lacking in confidence when presenting cases on ward rounds or speaking up for themselves. This extended to the educational relationship, with overseas doctors not necessarily used to asking for feedback and reflecting on their practice.

\footnotetext{
"The most striking thing to us, it's part of their cultural approach to the hierarchy of the hospital work...they are very subservient to everyone else. And...that's fine, but then they're open to abuse with that if they're constantly sort of being subservient ... they're almost open to getting slightly abused by people, so by senior members of staff who just kind of think, oh, they're a walkover". (ES3)
}

"I think they [overseas graduates] often seem to be lacking in confidence...speaking up and, you know, presenting patients on ward rounds, things like that...they sort of tend to be a bit in awe of us...I think it usually sorts itself out". (ES7)

Relational differences are embedded in practice, reflecting more fundamental elements of identity and worldview embodied in the way medicine is practiced. These differences are hard to address in simple informational terms. Participants felt that supportive and helpful multiprofessional teams had made their transition into the workplace easier by appreciating it would take time to learn.

\footnotetext{
"[Doctors and nurses] all understand what, where I come from... that I would need some sort of adjustment period just to familiarise myself with everything...they made some allowances for that, for a time period". (60b, EU)
}

\section{Discussion}

Interviews with overseas-qualified doctors at three points in their first year of working as an FY1 in the UK, triangulated by interviews with senior doctors working as educational supervisors, identified issues in a number of areas. Our analysis identified four main areas of challenge:

- Practical and logistical difficulties

- Gaps in declarative knowledge relevant to the UK, for example, use of equipment and drug names

- Structural differences, for example, organisational elements of working in the NHS

- Differences in the relational aspects of professional work and clinical care, stemming from training in different models of healthcare

Differences stemmed from different training cultures, and attitudes to elements of healthcare such as a patient-centred focus, and flatter hierarchies found in the UK. The transition was aided by previous experience of living and working in the UK, support from friends and family, and induction programmes specific to overseas doctors.

The four analytical themes identified have some similarity to the three elements of expatriate adjustment as introduced by Black; ${ }^{11}$ Work adjustment, General adjustment [cultural] and Interaction adjustment. Our analysis identifies a similar range of issues, but identifies greater overlap between work and non-work elements, and an explicit distinction between knowledge and work-based elements. This is perhaps due to greater complexity in the clinical workplace, where the nature of healthcare means that cultural factors are an intrinsic part of work.

Practical difficulties in immigration and physically settling in the UK may prevent doctors being able to take up their posts on time, having consequences for service delivery. Failures in communication have been identified as a cause of errors in clinical practice so gaps in knowledge of language and jargon may have serious consequences. Similarly lack of knowledge of care pathways and intra- 
organisational and inter-agency relationships may adversely affect care.

The findings of this study may indicate an underlying assumption within the NHS that overseas qualified doctors can function as effectively as UK-trained doctors without additional support in the transition to the UK workforce. However, it is possible that a lack of support with adjustment issues may, at least in part, explain the overrepresentation of overseas-qualified doctors in referrals to the GMC and NCAS. ${ }^{5-7}$ Beyond the safety concerns this raises, the cost to the NHS of investigations, exclusions and suspensions is large.

\section{Easing the transition}

Different approaches to addressing the challenges for overseas doctors may be identified. Different methods will be appropriate for addressing knowledge or work-based differences, and will be relevant at different stages in the doctors' transitions: before the doctor starts work, when they arrive and start work, and on-going into their practice. Possible interventions are identified below, with suggestions of the level of organisation within whose remit they fall. Different systems will have different specific components, but for the purposes of this discussion, three key players are identified: governmental departments (in the UK this will be the Department of Health for healthcare policy, and the Foreign Office for immigration policy, although other agencies are involved); the medical regulator (the GMC in the UK), and employers (NHS trusts, although Deaneries recruit directly for some training posts, including Foundation Programme).

It should be noted that many of these interventions will also be appropriate for aiding the transition of homecountry medical graduates to practice. The effectiveness of any support will also depend on the engagement of the individual doctor - some will find information and adapt without any support, some will require more support.

\section{Before moving to the UK}

Before the doctor starts work or moves to the UK, they will need to know the procedures for applying for posts, immigration requirements, housing immediately on arrival, etc. Our participants found that this information was not always available. For some the timescale of their appointment was an issue in this, with some employers recruiting overseas at short notice when training posts had not been filled by UK or EU graduates (as per European legislation).

Information can also be provided at this stage on any of the differences identified above. Priority should perhaps be given to those areas which do not require much contextual information, and/or embedding in experience. This may include glossaries of local vocabulary and use of equipment and drug names, which may be provided before starting work to enable familiarisation.

There are concerns that there may be insufficient checks on non-clinical knowledge when doctors move between 80 regulatory systems. In the UK, non-EU doctors must pass a series of clinical and language tests, such as the Professional and Linguistic Assessments Board (PLAB) test to register with the GMC. There is no such restriction on EU doctors, where cross-border movement of labour is legally established. The GMC is currently questioning the adequacy of this system in relation to patient safety.

The responsibility for information provision at this point is shared amongst all stakeholders. Government has responsibility for the healthcare policies that influence overseas recruitment and the immigration policies which enable it. The regulator can set standards for practice which employers must meet, whilst employers are responsible for direct recruitment and provision of local information.

\section{At the point of transition}

Some concepts which require more contextual knowledge and direct salience to be developed may be best introduced when the doctor has moved to the UK, and is starting work. In the UK NHS Trusts are required to provide an induction for all doctors, but only a few Trusts and Deaneries offer additional induction for international graduates. ${ }^{37}$ Several studies have indicated that a structured induction process is a useful way to help integrate doctors during the transition to the NHS. ${ }^{24,38-43}$ Couser $^{43}$ suggests induction programmes need to be interactive, with small group work and role play relating activities to the workplace, but it is important to be mindful of trainees' different learning styles which may be related to cultural preferences. While the provision of induction will necessarily be the responsibility of employers, the content of such induction can be specified by government or regulator policy. It should be a concern that not all doctors were able to attend even their general induction and shadowing periods due to visa issues, and both employers' recruitment strategies, and the immigration framework should be reviewed to see how such problems can be avoided. The GMC in their report on the State of Medical Education and Practice in the $\mathrm{UK}^{2}$ recognised that overseas doctors need support in the transition to the UK. An induction programme for new doctors to UK practice is to be piloted by the end of 2012 and will introduce doctors to the ethical and practical issues around medical practice in the $\mathrm{UK}^{32}$

\section{On-going workplace support}

Relational differences require the development of skilled interactions. While these may be practiced at induction, they will require most development when the new overseasqualified doctor is in post. To do this, they need to be attuned to the need to develop these skills, but the workplace, and the doctor's colleagues, should also provide support to incoming doctors and other professionals.

Possible approaches to providing support are specific mentoring and buddying schemes, where a new arrival is partnered by a senior to introduce them to the locality and the work. The mentor may also be overseas-qualified, not 
necessarily from the same country. Chen et al. ${ }^{19}$ found that intergenerational mentoring can help reduce isolation and discrimination because the mentor will have already had experience. Mentoring can lead to greater organisational knowledge and performance. ${ }^{44}$ General co-worker support can also lead to improved work adjustment, ${ }^{19,45}$ although the extent of support can vary with demographic variables such as ethnicity and gender. ${ }^{46}$

Educators and supervisors should also be aware of the different experiences and expectations of doctors from overseas, who may be less used to working in groups and more used to being a passive learner. ${ }^{43}$ Cultural awareness training programmes may develop these skills in all elements of the workforce. Such programmes and mentoring interventions will also be necessarily the responsibility of the employer, but as with other initiatives these should be guided and standardised by government and regulator.

\section{Conclusions}

The findings highlight that important differences are experienced when overseas-qualified doctors move to, and start work in, the UK. A failure to address these may leave both the doctor and patient vulnerable. Some of the challenges that have been highlighted are also experienced by overseas doctors in other countries, such as the USA, Canada and Australia. ${ }^{19}$

Suggested solutions address each stage of the doctors' transition - before coming to the UK, at the point at which they start work and on-going support when they are in their post. The responsibility for these may lie with government (developing policy), regulator (developing standards) and employer (implementing and ensuring fitness to practise). It is therefore essential that these bodies look seriously at what can be done to support incoming doctors, and aim to develop an integrated support framework for doctors whose contribution is essential to the delivery of healthcare, but who may be under-supported in crucial ways.

\section{Limitations}

The study has some limitations from the samples available at the time of recruitment. There was a lack of EU doctors coming to the UK to start FY1 training in the year of the study, meaning few could be recruited. The majority of these were from 'new' European countries which joined the EU from 2003. These may be different from more established, wealthier countries.

While the aim was to look at the breadth of the incoming population, the fact that the doctors were so disparate may have confounded some of their experiences, although the idiographic approach to analysis will mean individual views are not lost. Some differences may be more salient to some participants than others. There was some attrition of participants between initial and follow-up interviews due to a change in contact details or not being able to be interviewed within the study timescale. It is possible that some constituted a particular subgroup or had experiences that contributed to their not being able to take part in the research, but it was not possible to explore this within the study.

\section{Acknowledgements}

This project was funded by the ESRC Public Services Programme RES-153-25-0097. We also thank all participants in the research.

\section{Conflict of Interest}

The authors declare that they have no conflict of interest.

\section{References}

1.Clark PF, Stewart JB, Clark DA. The globalization of the labour market for health-care professionals. Int Labour Rev. 2006;145(1-2):37-64.

2.General Medical Council. The state of medical education and practice in the UK. 2011 [cited 23 August 2012]; Available from: http://www.gmcuk.org/publications/10586.asp.

3.BMA. Doctors new to the UK. 2013 [cited 16 February 2013]; Available from: http://bma.org.uk/practical-support-at-work/immigration/doctorsnew-to-the-uk/immigration-regulations.

4.General Medical Council. Annual report and accounts. 2008 [cited 14 September 2011]; Available from: http://www.gmc-uk.org/publications/ annual_reports/Annual_Report_and_Accounts_2008.pdf.

5. General Medical Council. Fitness to practise annual statistics 2008. 2009 [cited 16 September 2011]; Available from: http://www.gmcuk.org/publications/2009_annual_statistics.asp.

6. National Clinical Assessment Service. NCAS casework: the first eight years. 2009 [cited 24 November 2012]; Available from: http://www.ncas. nhs.uk/publications/.

7. National Clinical Assessment Service. Concerns about professional practice and associations with age, gender, place of qualification and ethnicity - 2009/10 data. 2011 [cited 12 August 2012]; Available from: www.ncas.nhs.uk/publications/statistics.

8. Slowther A, Lewando Hundt GA, Purkis J, Taylor R. Experiences of nonUK-qualified doctors working within the UK regulatory framework: a qualitative study. J R Soc Med. 2012;105:157-65.

9. RAND. International comparison of ten medical regulatory systems. 2009 [cited 4 February 2013]; Available from: http://www.gmc-uk.org/about /research/research_commissioned_2.asp.

10. Andreason AW. Expatriate adjustment to foreign assignment. International Journal of Commerce and Management. 2003;13:42-60.

11. Black JS. Work role transitions: a study of American expatriate managers in Japan. J Int Bus Studs. 1988;19:277-94.

12. Black J, Stephens G. The influence of the spouse on American expatriate adjustment and intent to stay in Pacific Rim overseas assignments. J Manage. 1989;154:529-44.

13. Harrison DA, Shaffer MA. Mapping the criterion space for expatriate success: task - and relationship-based performance, effort and adaptation. International Journal of Human Resource Management. 2005;16(8):145474.

14. Sladden J. Overseas doctors' health. 2006 [cited 16 February 2013]; Available from: http://careers.bmj.com/careers/advice/view-article. html?id=1403.

15. Ezsias A. Refugee doctors face enormous difficulties. BMJ. 1998;316:1095.1.

16. Hall P, Keely E, Dojeiji S, Byszewski A, Marks M. Communication skills, cultural challenges and individual support: challenges of international medical graduates in a Canadian healthcare environment. Med Teach. 2004;26(2):120-5.

17. Spike NA. International medical graduates: the Australian perspective. Acad Med. 2006;81(9):842-6.

18. Pilotto LS, Duncan, GF, Anderson-Wurf J. Issues for clinicians training international medical graduates: a systematic review. Med J Aust. 2007;187(4): 225-8. 
19. Chen PGC, Curry LA, Bernheim, SM, Berg D, Gozu A, Nunez-Smith M. Professional challenges of non-US-born international medical graduates and recommendations for support during residency training. Acad Med. 2011;86(11):1383-8.

20. Cole-Kelly K. Cultures engaging cultures: international medical graduates training in the United States. Fam Med. 1994;26:618-24.

21. Majumdar B, Keystone JS, Cuttress LA. Cultural sensitivity training among foreign medical graduates. Med Educ. 1999;33(3):179-84

22. Stewart, E. A bitter pill to swallow: obstacles facing refugee and overseas doctors in the UK. Working paper 96, UNHCR - evaluation and policy analysis unit. 2003 [cited 16 February 2013]; Available from: http://www.unhcr.org/refworld/topic,4565c22553,4565c25f695,4ff2ae962,0, RESEARCH,html.

23. Bates J, Andrew R. Untangling the roots of some IMGs poor academic performance. Acad Med. 2001;76(1):43-6.

24. Bregazzi R, Harrison J. From Spain to county Durham; experience of cross-cultural general practice recruitment. Educ Prim Care. 2005;16(3): 268-74.

25. Mahajan J, Stark P. Barriers to education of overseas doctors in paediatrics: a qualitative study in South Yorkshire. Arch Dis Child. 2007;92 (3):219-23.

26. Gould M. Eastern European doctors and the NHS - everyone wins? 2005 [cited 13 February 2013]; Available from: http://careers.bmj.com/ careers/advice/view-article.html?id=859.

27. Fiscella K, Roman-Diaz M, Lue B-H, Botelho R, Frankel R. 'Being a foreigner, I may be punished if I make a small mistake': assessing transcultural experiences in caring for patients. Fam Pract. 1997;14(2):112-6.

28. Hawken S. Overseas-trained doctors' evaluation of a New Zealand course in professional development. N Z Med J. 2005 Jul 29;118 (1219):U1584

29. Kramer MN. The educational needs of international medical graduates in psychiatric residencies. Acad Psychiatry. 2005;29(3):322-3.

30. Stewart M, Brown JB, Weston WW, McWhinney IR, McWilliam CL, Freeman TR. Patient-centered medicine: transforming the clinical method. 2nd ed. Oxon: Radcliffe Medical Press Limited; 2003.

31. Hamilton J. The collaborative model of doctor-patient consultation - Is it always culturally appropriate? What do doctors and patients need to know to make it work in intercultural contexts? Med Teach. 2009;31(2):163-5.

32. Carter S. GMC to pilot an induction programme for doctors new to UK practice. 2012 [cited 16 February 2013]; Available from: http://careers.bmj.com/careers/advice/view-article.html?id=20008142.
33. Illing J, Kergon C, Morrow G, Burford B. The experiences of UK, EU and non-EU medical gradu-ates making the transition to the UK workplace: full research report. ESRC end of award report, RES-153-25-0097. 2009 [cited 11 June 2010]; Available from: http://www.esrc.ac.uk/myesrc/grants/RES-153-25-0097/read.

34. Charmaz K. Grounded theory: objectivist and constructivist methods. In: Denzin DK, Lincoln YS, editors. Strategies of qualitative inquiry. California: Sage Publications, Inc.; 2003.249-91.

35. QSR Software. Using NVivo in qualitative research. Melbourne, Australia: QSR international; 2002.

36. Varpio L, Hall P, Lingard L, Schryer CF. Interprofessional communication and medical error: a reframing of research questions and approaches. Acad Med. 2008;83 S76-S81.

37. Slowther A, Lewando Hundt G, Taylor R, Purkis, J. Non UK qualified doctors and good medical practice: the experience of working within a different professional framework. 2009 [cited 3 September 2010]; Available from: http://www.gmc-uk.org/FINAL_GMC_Warwick_Report.pdf_253922 30.pdf.

38. Rich AJ. An induction programme for first-appointment overseas doctors. Med Teach. 1998;20(5):473-5.

39. Ong Y-L, McFadden G, Gayen A. Induction for overseas qualified doctors. Hosp Med. 2002;63(9):558-60.

40. Ballard K, Robinson S, Laurence P. Why do GPs from France choose to work in London practices? A qualitative study. Brit J Gen Pract. 2004;54 (507):747-52.

41. Rajesh U, Nagrani R. Overseas registrars training in obstetrics and gynaecology in Wales - a questionnaire survey. J Obstet Gynaecol. 2004;24(1):78-80.

42. Shah M, Goswami S, Singh G, Brown R. Overseas consultant psychiatrists moving into the NHS: initial experience. Psychiatr Bull R Coll Psychiatr. 2006;30(6):228-9.

43. Couser $\mathrm{G}$. Twelve tips for developing training programs for international medical graduates. Med Teach. 2007;29(5):427-30.

44. Carraher SM, Sullivan SE, Crocitto MM. Mentoring across global boundaries: an empirical examination of home- and host-country mentors on expatriate career outcomes. J Int Bus Stud. 2008;39:1310-26.

45. Aryee S, Stone RJ. Work experiences, work adjustment and psychological well-being of expatriate employees in Hong Kong. The International Journal of Human Resource Management. 1996;7(1):150-64.

46. Olsen JE, Martins LL. The effects of expatriate demographic characteristics on adjustment: a social identity approach. Human Resource Management. 2009;48(2):311-28. 